\title{
A highly stretchable, helical copper nanowire conductor exhibiting a stretchability of $700 \%$
}

\author{
Yulim Won ${ }^{1}$, Areum Kim ${ }^{1}$, Wooseok Yang ${ }^{1}$, Sunho Jeong ${ }^{2}$ and Jooho Moon ${ }^{1}$ \\ Recent advances in unconventional foldable and stretchable electronics have forged a new field in electronics. However, \\ traditional conducting metal oxides and metal thin films are inappropriate as electrodes for stretchable devices because they \\ are vulnerable to tensile strain as well as bending strain. In this study, we describe the fabrication of annealing-free, copper \\ nanowire (CuNW)-based stretchable electrodes using an inexpensive metal source through a simple and scalable process at \\ low temperature without a vacuum. We also introduce a reversible and extremely stretchable (up to $700 \%$ of strain) helical, \\ CuNW-based conducting spring, which has not been previously used for stretchable electrodes.
}

NPG Asia Materials (2014) 6, e132; doi:10.1038/am.2014.88; published online 26 September 2014

\section{INTRODUCTION}

Recent advances in unconventional foldable and stretchable electronics show great potential for future wearable applications, including smart skins, electronic eye-type imagers, skin-like pressure sensors, electronic textiles and muscle-like soft actuators. ${ }^{1-5}$ Most of these applications require sufficient elasticity for bending, stretching, twisting and deformation into complex, non-planar shapes while maintaining good electrical properties and reliability. Stretchability is the most crucial property for the development of next-generation wearable devices. To date, two primary strategies have been suggested for the fabrication of stretchable electronic parts apart from complex lithography-based approaches that can shape inorganic conductive materials or metals into buckled geometries, including island-bridge systems. ${ }^{6-9}$ The first strategy is the inclusion of micro- or nano-scale conductive carbon materials into elastic polymer matrices. ${ }^{1,10-13}$ For example, carbon-based materials, such as carbon nanotubes and graphene, have been extensively researched for stretchable/foldable conductors. Ma et al. ${ }^{13}$ fabricated helical ribbon-structured composites composed of carbon nanotubes and Ag flakes using a shape-memory polymer, demonstrating stable resistivity up to a strain of $600 \%$. However, the application of these materials in large-area, integrated devices may be restricted by their relatively poor electrical properties and their high cost. The second strategy is to use highly conductive metallic nanostructures, such as rectangular-patterned gold nanosheets $^{14}$ (strain $(\varepsilon)=100 \%$ on Ecoflex substrate) or silver nanoparticles embedded in composite materials ${ }^{15}(\varepsilon=140 \%$ on elastomeric rubber fiber).

Recently, networks of one-dimensional metal nanowires, accompanied by a simple, scalable process (for example, solution-phase mixing and coating) have emerged as an alternative approach to facilitate conductive materials with superior stretchability. ${ }^{16,17}$ In weblike nano-structured metal geometries, the strain applied by a mechanical stretching stress is accommodated by in-plane rotations and distortions of the nanowire networks. Thus, unconventional nanostructures, which are generated by the spatial displacement between nanowires with a high aspect ratio, can maintain their continuous network structures, which act as an electrical charge path, while releasing the strain accumulated during stretching. As proof of this concept, there have been a limited number of reports on silver nanowire (AgNW)-based stretchable electrodes ${ }^{17-19}$ and AgNWcarbon composite-based hybrid electrodes. ${ }^{20,21}$ However, for practical applications, inexpensive, nanowire-structured $\mathrm{Cu}$ metal materials are suggested as a new, cost-effective approach to creating stretchable electrodes, replacing the high-cost noble metals that hinder the broad adoption of these appealing approaches.

The preparation of one-dimensional $\mathrm{Cu}$ nanomaterials suitable for stretchable electronics is significantly demanding, owing to the requirement of a high aspect ratio for stretchability and high electrical conductivity, as well as surface oxidation-free phase purity with a high surface-to-volume structure. Recently, electrospun copper nanofiberbased web structures have been developed without the need for hightemperature reductive annealing;, ${ }^{22,23}$ however, the mechanical/ electrical stretchability of the $\mathrm{Cu}$ nanofibers has not been investigated in sufficient detail for these materials to be applied to stretchable electronics. Similar to the case of $\mathrm{Ag}$ nanowires, copper nanowires (CuNW), which are synthesized in the liquid phase, have been used as stretchable electrodes owing to their reversible sliding characteristics. ${ }^{24}$ However, to date, the strain stability and effect of the

\footnotetext{
${ }^{1}$ Department of Materials Science and Engineering, Yonsei University, Seoul, Republic of Korea and ${ }^{2}$ Advanced Materials Division, Korea Research Institute of Chemical Technology, Daejeon, Republic of Korea

Correspondence: Dr S Jeong, Advanced Materials Division, Korea Research Institute of Chemical Technology, 19 Sinseongno, Yuseong-gu, Daejeon 305-600, Republic of Korea. E-mail: sjeong@krict.re.kr

or Professor J Moon, Department of Materials Science and Engineering, Yonsei University, 50 Yonsei-ro Seodaemun-gu, Seoul 120-749, Republic of Korea.

E-mail: jmoon@yonsei.ac.kr
}

Received 29 April 2014; revised 31 July 2014; accepted 31 July 2014 
structure-tailored substrate have been not studied for CuNW-based network films. Herein, we describe the fabrication of an annealingfree, phase-pure, CuNW-based stretchable electrode by way of a simple, scalable, synthetic process combined with a chemical posttreatment in an ambient atmosphere. This cost-effective new class of $\mathrm{Cu}$ materials possesses extreme stretchability of up to $700 \%$ due to the introduction of an unconventional helical-structured substrate, which has not been previously applied for metallic stretchable electrodes.

\section{EXPERIMENTAL PROCEDURE}

\section{Materials}

Copper (II) chloride dihydrate, 1-hexadecylamine, dextrose and DL-lactic acid were purchased from Sigma-Aldrich (St Louis, MO, USA). Isopropyl alcohol (IPA) and n-hexane were obtained from Duksan Pure Chemical (Ansan-si, Gyeonggi-do, Korea). The mixed cellulose ester membrane filter (a white filter with a diameter of $25 \mathrm{~mm}$ and a pore size of $1 \mu \mathrm{m}$ in diameter) was purchased from ADVANTEC (Bunkyo-ku, Tokyo, Otowa, Japan). Parylene-C dimer (Dix-C) was supplied by KISCO (Tokyo, Japan). All of the chemicals were used as received without further purification.

\section{Synthesis of CuNWs}

The CuNWs were synthesized using a previously published report, with slight modifications. ${ }^{25}$ To synthesize thin and long CuNWs, $50 \mathrm{mg} \mathrm{CuCl}_{2}{ }_{2} \mathrm{H}_{2} \mathrm{O}$, $280 \mathrm{mg}$ hexadecylamine and $100 \mathrm{mg}$ dextrose were dissolved in $20 \mathrm{ml}$ of water at room temperature. The final solution was placed in a vial and aged at room temperature for $12 \mathrm{~h}$ with magnetic stirring. After the vial was capped with paraffin tape, the capped vial was heated in an oil bath at $100{ }^{\circ} \mathrm{C}$ for $6 \mathrm{~h}$. Upon complete reaction, the solution had a red-brown color. The synthesized nanowires were washed three times with hexane and IPA (hexane:IPA $=2: 1$ ). The purified CuNWs were then stored in IPA. After nanowire aggregation, the sediment was removed by centrifugation $(500 \mathrm{rpm}$ for $3 \mathrm{~min}$ ) and the CuNW dispersion was treated with lactic acid for $10 \mathrm{~s}$. Longer lactic acid treatment times resulted in morphological changes in the CuNWs due to etching. ${ }^{26}$ The lactic acid was added to remove the residual organics and copper oxide.

\section{Determination of the concentration of the $\mathrm{CuNW}$ dispersion}

The concentration of the CuNW dispersion was determined by inductively coupled plasma optical emission spectrometry (ICP-OES, OPTIMA 8300, Perkin Elmer, Waltham, MA, USA) analysis. To determine the concentration of the CuNW dispersion used for filtration, a set volume of the dispersion $(3 \mathrm{ml})$ was dissolved in nitric acid $(3 \mathrm{ml})$, followed by evaporation at $70{ }^{\circ} \mathrm{C}$. The dried substance was then further re-dissolved in a mixture of deionized water $(1 \mathrm{ml})$, nitric acid $(3 \mathrm{ml})$, and hydrogen peroxide $(1 \mathrm{ml})$.

\section{Fabrication of helical-structured polydimethylsiloxane (PDMS)} substrate and transferring of the CuNWs

A screw was used as a mold for the helical structure. To fill the PDMS in the furrows of the screw without any bubbles, a straw was used that fit perfectly with the screw. The PDMS was filled in the straw and the air bubbles were removed. The screw was inserted into the PDMS-filled straw by turning the screw. The PDMS was then cured at $70^{\circ} \mathrm{C}$ in an oven overnight. After curing, the straw was removed and the helical-shaped PDMS was recovered by peeling it from the screw. To remove the uncured PDMS contaminants from the surface, the helical-structured PDMS was washed with IPA and then dried in an oven. Finally, the CuNW network was transferred onto this substrate by rolling the PDMS helix on the CuNW film.

\section{Structural, optical and electrical characterization}

Field-emission scanning electron microscopy (JEOL-6701F, JEOL Inc. Peabody, MA, USA) was used to characterize the structural properties of the nanostructured thin films. The sheet resistance values $\left(R_{\text {sh }}\right)$ of the CuNW samples were measured by a four-point probe system (RS8, BEGA Technologies, Gyeonggi-do, Korea). The resistance was reported as an average value obtained after at least 10 measurements. The mechanical stretchability of the planar and helical-structured CuNW/PDMS electrodes was determined as a function of the number of stretching cycles by repeatedly stretching the electrodes with a two-point stretching device. The resistance values were

a
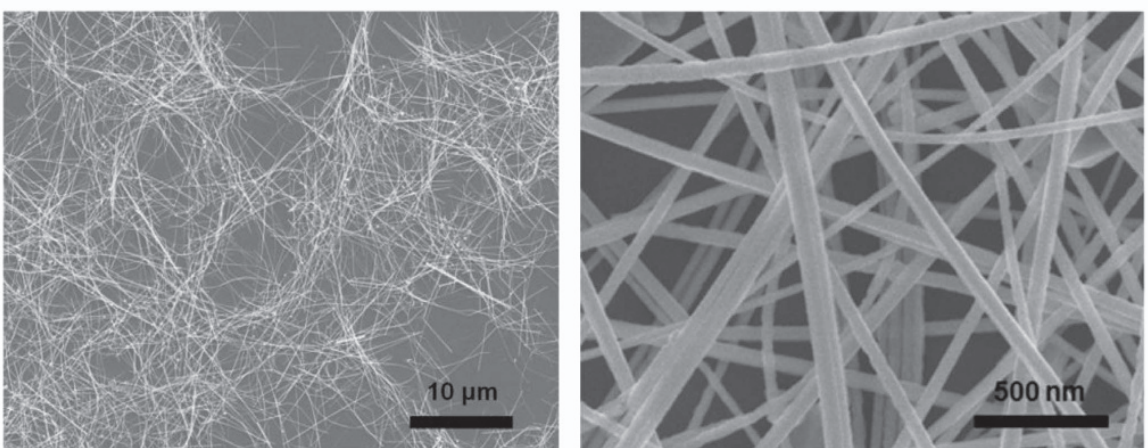

b
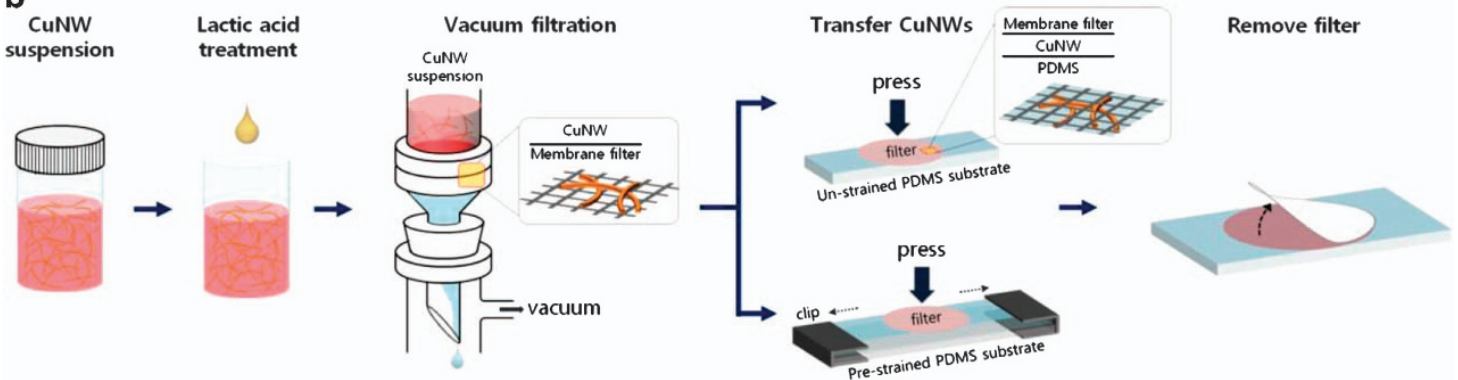

Figure 1 (a) FESEM image of the CuNWs after chemical treatment. (b) Schematic representation of the preparation of the stretchable CuNW electrodes on both unstrained planar PDMS and pre-strained PDMS substrates, both ends of which were elongated by clips. 
a

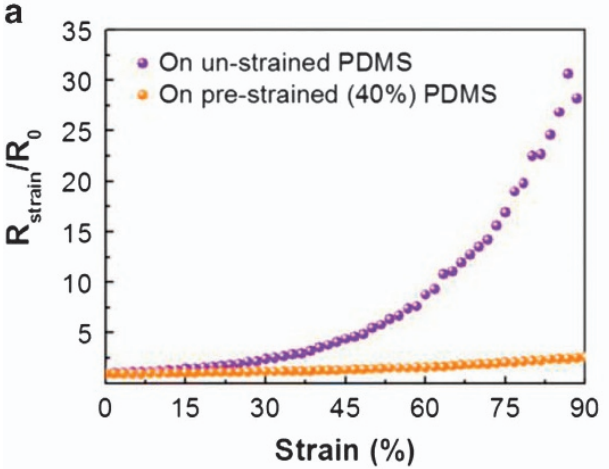

d

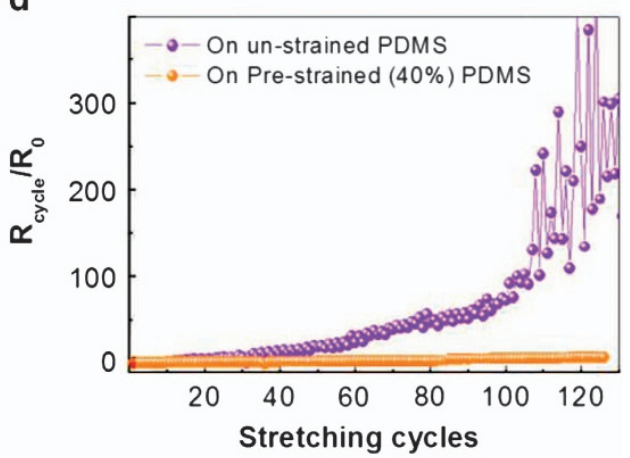

b
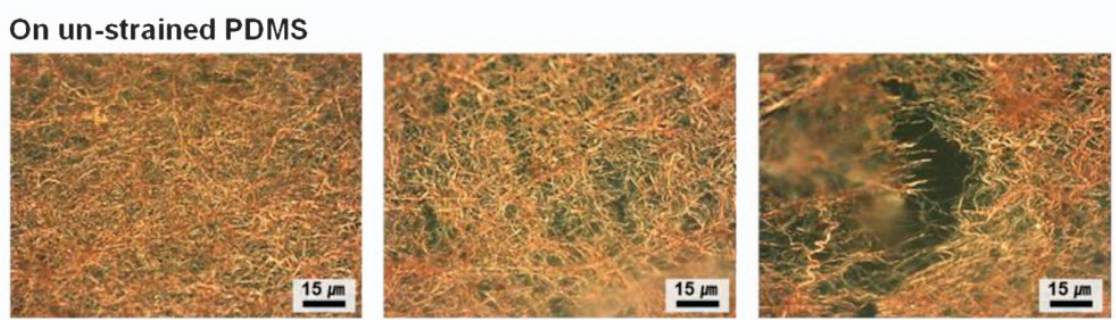

On pre-strained PDMS
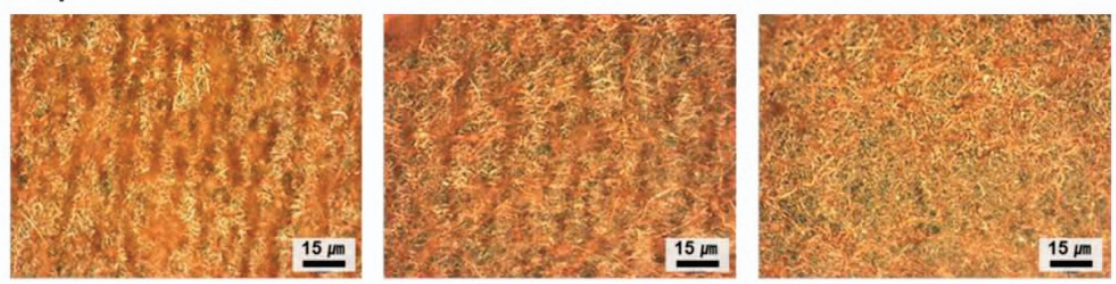

$\varepsilon=0 \%$

$\varepsilon=30 \%$

$\varepsilon=50 \%$

C

On un-strained PDMS

On pre-strained PDMS
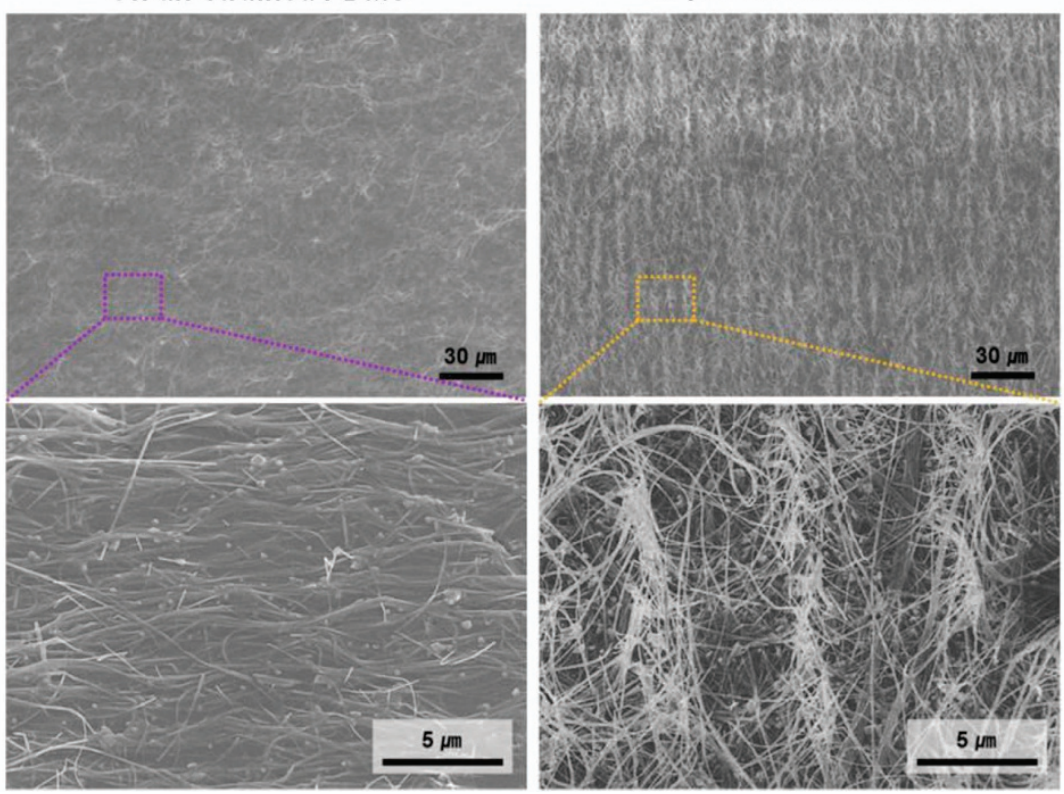

Figure 2 (a) Relative resistance change under various strains for stretchable CuNW electrodes fabricated on the planar and pre-strained PDMS substrates. (b) Optical images and (c) FESEM images of CuNW networks during stretching. (d) Relative resistance variations of the CuNW electrodes fabricated on both unstrained and pre-strained substrates as a function of repeated stretching cycles. 
measured on the flattened films after a given number of stretching cycles using a four-point probe. For the stretching test, eutectic gallium-indium liquid metal was painted on both ends of the CuNW film. The stretchable CuNW conductor was clamped by two fixtures and the electrical resistance was measured under various tensile strains. The current-voltage $(I-V)$ measurements were made using an Agilent 4155C analyzer (Agilent, Santa Clara, CA, USA).

\section{RESULTS AND DISCUSSION}

The CuNWs were synthesized through the chemical reaction of copper chloride in an aqueous solvent in the presence of a capping agent, hexadecylamine, in a well-controlled manner (see Experimental section for details). The as-synthesized CuNWs had an average diameter of $\sim 66 \pm 17 \mathrm{~nm}$ and an average length of $>50 \mu \mathrm{m}$ (Figure 1a). Long nanowires with a high aspect ratio are required in order to achieve both mechanical stretchability and electrical conductivity in the nanowire-based web structures, because they enable fully inter-networked, highly conductive pathways even with low nanowire density, which releases the stress during mechanical elongation. ${ }^{24}$ The nanowires were dispersed in IPA at a concentration of $203.8 \mathrm{mg} \mathrm{ml}^{-1}$ and subsequently chemically treated with lactic acid to completely remove both the surface oxide/hydroxide and the chemisorbed organic capping molecules, which diminished the electrical conductivity of the CuNW network films. ${ }^{26}$ The chemical transformation into phase-pure $\mathrm{Cu}$ nanowires through a simple wet treatment facilitated the fabrication of highly conductive $\mathrm{Cu}$ nanomaterials at room temperature without the incorporation of toxic chemical environments, unlike the previously reported methods. ${ }^{27,28}$ Notably, for AgNWs prepared using sophisticated procedure, an annealing process at slightly elevated temperatures ranging from $80-220^{\circ} \mathrm{C}$ is highly recommended for inducing nano-welding between neighboring nanowires owing to the presence of organic capping molecules. In some cases, this can limit the integration of thermally vulnerable functional moieties. As shown in Figure $1 \mathrm{~b}$, the conductive CuNW network films were prepared by vacuum filtration, followed by transfer printing onto a PDMS substrate, which is most widely used as an elastic polymer matrix.

The vacuum-deposited $\mathrm{Cu}$ thin films prepared on the PDMS substrate as a control sample suffered from mechanical rupture at a tensile strain of 30\% (Supplementary Figure S1). This implies that the non-structurally engineered $\mathrm{Cu}$ material does not afford relieve the applied tensile strain, even with the ductile property of the $\mathrm{Cu}$ itself. Cracks developed on the surface of the films perpendicular to the stretching direction. The CuNW network film transferred onto the PDMS substrate showed improved tensile strain tolerance (Figure 2a), but the resistance of the CuNW film was doubled under a strain of

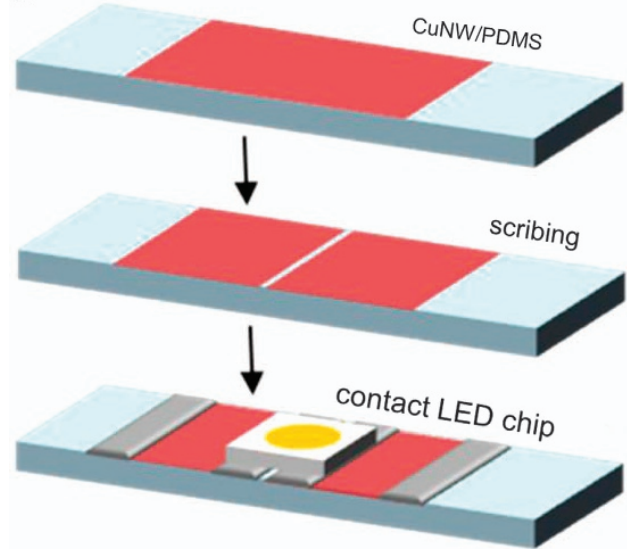

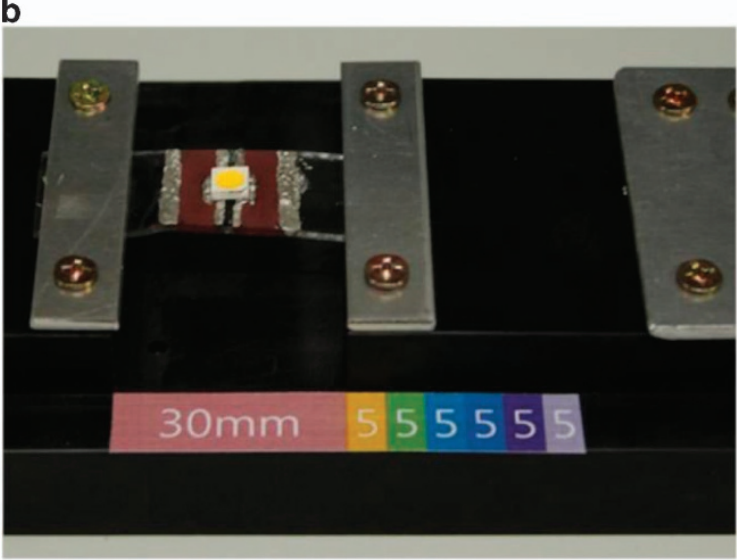

C

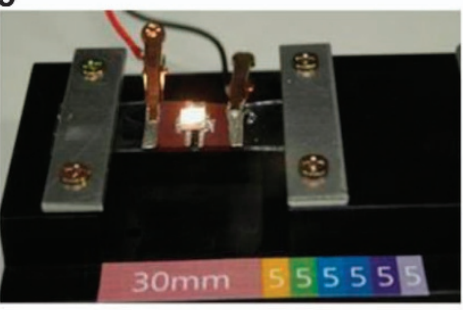

$\mathcal{E}=16.7 \%$

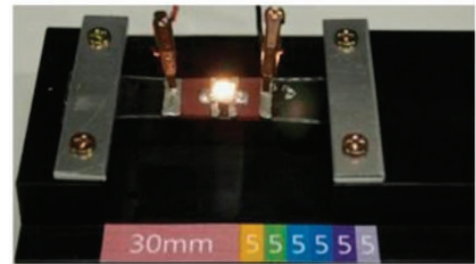

$\mathcal{E}=66.7 \%$

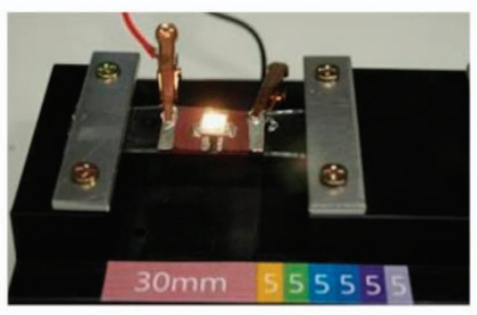

$\mathcal{E}=33.3 \%$

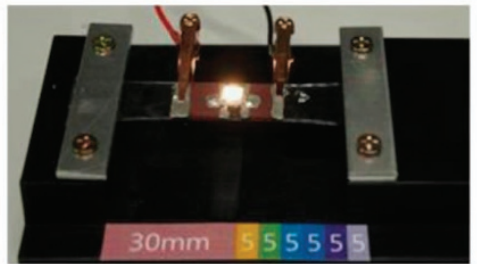

$\mathcal{E}=83.3 \%$

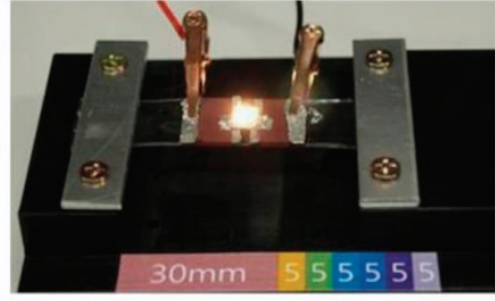

$\mathcal{E}=\mathbf{5 0} \%$

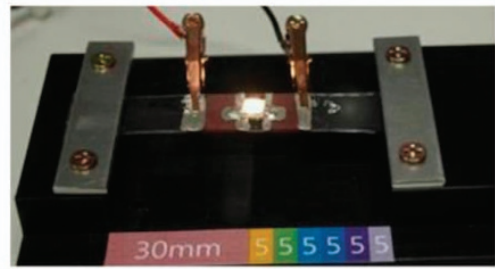

$\mathcal{E}=100 \%$

Figure 3 (a) Schematic representation of the LED circuit preparation. (b) A photograph of the LED circuit attached to the stretchable CuNW/PDMS electrode. (c) A photograph of the LED light on the stretchable CuNW electrode with increasing tensile strain. 
$25 \%$ and significantly increased with further strain, which is not acceptable for commercialization (30-40\% strains). ${ }^{15}$ As for the fieldemission scanning electron microscopy and optical microscopy images, the surface of the CuNW film fabricated on the PDMS substrate was torn off in the stretching direction when the applied tensile strain was $>30 \%$ (Figures $2 \mathrm{~b}$ and $\mathrm{c}$ ). However, it should be noted that the stretching of the annealing-free, phase-pure, long CuNW film was almost comparable to that of the AgNW films welded at the nano-scale by annealing at $220^{\circ} \mathrm{C} .{ }^{19}$ To further improve their stretchability, the CuNW networks were transferred onto a $40 \%$ pre-strained PDMS substrate. The sheet resistance was $6-12 \Omega /$ sq. The optical images and field-emission scanning electron microscopy images showed the wrinkled structure of the CuNW film after the strain was removed from the PDMS substrate (Figures $2 b$ and $c$ ). Three-dimensional confocal images also clearly revealed the wrinkled structure of the CuNWs (Supplementary Figure S2). This wrinkled structure behaves similarly to the buckling structures that have been reported for Si nanoribbons, ${ }^{29}$ facilitating effective extension of the CuNW networks at high strains of over $40 \%$. As shown in Figure $2 \mathrm{~b}$, the creased surface was folded without tearing at $\varepsilon=50 \%$, ensuring a substrate pre-strain effect on the $\mathrm{Cu}$ nanowire stretching. The resistance of CuNW film on the pre-strained PDMS increased slightly to an $R_{\text {strain }} / R_{o}$ of 2.5 , where $R_{o}$ and $R_{\text {strain }}$ are the resistance values measured before and after stretching, respectively, even when stretched to $\varepsilon=90 \%$ (Figure 2a). This low sheet resistance, which is a level at which general optoelectronic devices have been successfully applied, together with the high stretchability, were achieved in the metallic $\mathrm{Cu}$ nanostructures through the synthesis of long $\mathrm{Cu}$ nanowires using the room-temperature lactic acid-chemical treatment and the adoption of a pre-strained elastomeric substrate.

To examine the reversible stretching properties of the CuNW networks, we tested their performance as a function of repeated stretching cycles at a tensile strain of $30 \%$ (Figure 2d). For CuNW films fabricated on an unstrained PDMS substrate, the resistance was partially recovered release of the strain. The resistance increased gradually with repeated stretching cycles. On the other hand, the resistance of the CuNW on the pre-strained PDMS did not double over 130 cycles. As a demonstration of a stretchable device using CuNW electrodes, a light-emitting diode circuit was attached on a stretchable CuNW film transferred onto pre-strained PDMS with a small amount of silver paste and eutectic gallium-indium (Figures 3a,b). When the CuNW electrode was stretched, the lightemitting diode operated well, even under a tensile strain greater than $100 \%$ (Figure 3c). a
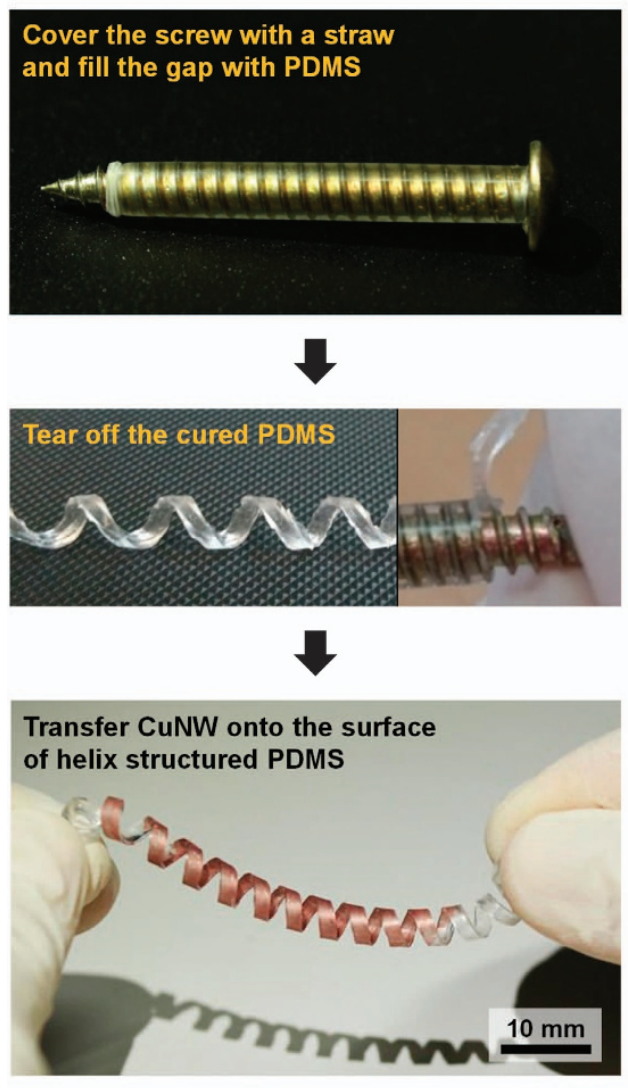

b
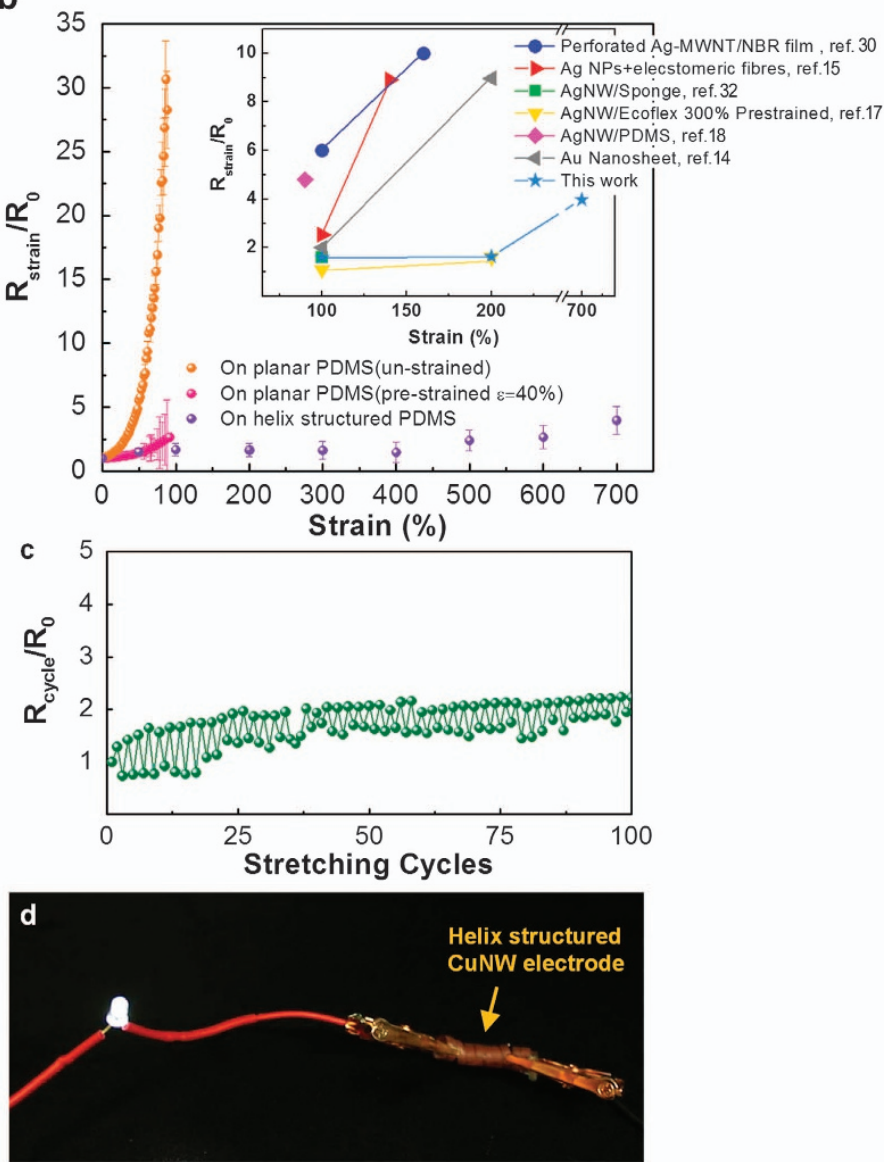

Figure 4 (a) Photographs of the fabrication process for the helical-structured CuNW electrodes. (b) Relative resistance variation as a function of strain for stretchable CuNW electrodes fabricated on both the planar and the helical-structured PDMS substrates. Both substrates were maintained under unstrained conditions while transferring the CuNW network films. (c) Relative resistance change of the CuNW electrodes fabricated on the helical-structured PDMS substrate during repeated stretching cycles at $\varepsilon=100 \%$. (d) A photograph of the LED light on a helical-structured CuNW electrode (see Supplementary movie S1 for the operation of this conducting spring). 
Next, we modified the matrix structure to further enhance the stretchability of the conductors. A simple variation in the matrix morphology provided material properties comparable to the previous approaches. ${ }^{30-32}$ Jang et al. ${ }^{31}$ reported that two-dimensional platinum nano-mesh materials prepared using an anodic aluminum oxide template improve the stretchability of Pt films on a PDMS substrate to $\varepsilon=20 \%$. Ge et al. demonstrated that the three-dimensional structure of an AgNW-based binary network design, in which AgNWs were infiltrated into a sponge-like PDMS skeleton, exhibits outstanding stretchability $(\varepsilon=140 \%) .{ }^{32}$ These findings indicate that the morphological structure has an important role in imparting mechanical stretchability beyond the inherent limitations of mechanical elongation. In particular, the structures that are modified into a three-dimensional geometry, rather than into a two-dimensional geometry, are beneficial for accommodating extreme strains. For example, a simple 2-mm-thick PDMS film can only be stretched up to $60 \%$ strain, ${ }^{3}$ but if the PDMS is shaped into a helical structure, exceptional stretchability can be obtained. Helical structures are found in many man-made objects, such as telephone cords and springs, as well as in nature, for example, deoxyribonucleic acid or climbing plants. Spring-like carbon nanotube yarn-shaped conductors with a high stretchability of up to $285 \%$ were previously reported. ${ }^{33}$ In this study, we describe a novel, highly stretchable and reversible, helicalstructured CuNW network conductor with significantly improved mechanical and electrical properties.

The helical-structured PDMS substrate was fabricated using a screw as a template. As shown in Figure 4a, the screw was inserted into a straw of slightly larger diameter. The PDMS precursor liquid was infiltrated into the gap between the screw and the straw. After curing, a helical-shaped PDMS substrate was obtained. The CuNW network was then transferred onto this substrate. Surprisingly, compared with the CuNW network transferred onto a planar PDMS substrate, the stretchability of the helix-shaped CuNW conductor was dramatically improved without pre-straining (Supplementary Figure S3). Although the relative resistance change $\left(\mathrm{R}_{\text {strain }} / \mathrm{R}_{\mathrm{o}}\right)$ of the CuNW films on planar PDMS increased by a factor of 30 at $100 \%$ elongation, the relative resistance change of the CuNW film on the helical-structured PDMS was only 3.9 at $\varepsilon=700 \%$, as shown in Figure $4 \mathrm{~b}$. The inset shows other results reported in the literature for highly stretchable electrodes. Ag nanoparticles and Ag nanowires were recently suggested as highly conductive metal moieties that can be implemented in elastomeric matrixes. When combined with elastomeric skeletons, including a sponge, ${ }^{32}$ PDMS, ${ }^{18}$ Ecoflex,${ }^{17}$ fiber, ${ }^{15}$ or carbon nanotube-embedded polymer, ${ }^{30}$ they exhibited superior durability at a strain of $100 \%$. However, the resistance significantly increased from 100 to $200 \%$. The resistance and stretchability at strains $>200 \%$ has not been reported to date. This can be attributed to the inherent stretching limit of elastomeric matrices and in turn, the structural collapse of the metallic constituents inside them. A highly stable electrode at a strain of $200 \%$ was achieved only with a $300 \%$-pre-strained elastomeric substrate. ${ }^{17}$ These comparative results indicate that helical elastomer-based CuNW films will be suitable materials for extremely stretchable electrodes. These stretchable electrodes will inspire the development of unconventional electronic systems, although there are still issues for in terms of scale-down and device integration for practical applications. In addition, the cost-effectiveness of CuNWs is one critical advantage over conventional, expensive AgNWs. The CuNWs also provide superior electrical/morphological properties compared with onedimensional metallic nanowires. As shown in Figure $4 \mathrm{c}$, the helical-structured CuNW conductor showed stable and reversible stretchability during repeated stretching cycles at $\varepsilon=100 \%$. As visually shown in Figure 4d and the Supplementary movie S1, the repeated operation of an light-emitting diode circuit mounted on the helical stretchable CuNW spring was successfully demonstrated.

\section{CONCLUSIONS}

We introduced CuNWs as stretchable, conductive building block materials that can be compatible with an extremely extendable, 3-dimensional-structured PDMS matrix to facilitate high stretchability of $700 \%$ without degrading the electrical resistance. Highly conductive, elongated 1-dimensional $\mathrm{Cu}$ nanowires were synthesized using a wet chemical process, followed by a room-temperature lactic acidchemical treatment that removed both the surface oxide and the thick surface capping layer. In addition to the basic studies on the effects of a pre-strained elastomeric substrate, the newly suggested helical conductor exhibited unprecedented stretchability of $700 \%$ with a resistance change of only 3.9, even when $\mathrm{Cu}$ nanowires were used instead of Ag nanowires. This methodology could pave the way for practical applications in various stretchable devices by providing better electrical/mechanical properties and cost-effectiveness.

\section{CONFLICT OF INTEREST}

The authors declare no conflict of interest.

\section{ACKNOWLEDGEMENTS}

This work was supported by a National Research Foundation (NRF) of Korea grant funded by the Korean government (MSIP) (No. 2012R1A3A2026417). This work was also partially supported by the third stage of the Brain Korea 21 Plus project.

1 Yamada, T., Hayamizu, Y., Yamamoto, Y., Yomogida, Y., Izadi-Najafabadi, A. Futaba, D. N. \& Hata, K. A stretchable carbon nanotube strain sensor for humanmotion detection. Nat. Nanotechnol. 6, 296 (2011).

2 Sekitani, T., Nakajima, H., Maeda, H., Fukushima, T., Aida, T., Hata, K. \& Someya, T. Stretchable active-matrix organic light-emitting diode display using printable elastic conductors. Nat. Mater. 8, 494 (2009).

3 Brosteaux, D., Axisa, F., Gonzalez, M. \& Vanfleteren, J. Design and Fabrication of Elastic Interconnections for Stretchable Electronic Circuits. IEEE Electon Device Lett. 28, 552 (2007).

4 Lipomi, D. J., Vosgueritchian, M., Tee, B. C., Hellstrom, S. L., Lee, J. A., Fox, C. H. \& Bao, Z. Skin-like pressure and strain sensors based on transparent elastic films of carbon nanotubes. Nat. Nanotechnol. 6, 788 (2011).

5 Gonzalez, M., Axisa, F., Bulcke, M. V., Brosteaux, D., Vandevelde, B. \& Vanfleteren, J. Skin-like pressure and strain sensors based on transparent elastic films of carbon nanotubes. Microelectro. Reliab. 48, 825 (2008).

6 Kim, D. H. \& Rogers, J. A. Stretchable electronics: materials strategies and devices. Adv. Mater. 20, 4887 (2008).

7 Rogers, J. A., Someya, T. \& Huang, Y. Materials and mechanics for stretchable electronics. Science 327, 1603 (2010).

8 Ko, H. C., Stoykovich, M. P., Song, J., Malyarchuk, V., Choi, W. M., Yu, C., Geddes, J. B. III, Xiao, J., Wang, S., Huang, Y. \& Rogers, J. A. A hemispherical electronic eye camera based on compressible silicon optoelectronics. Nautre 454, 748 (2008).

9 Kim, D., Song, J., Choi, W. M., Kim, H., Kim, R., Liu, Z., Huang, Y. Y., Hwang, K. Zhang, Y. \& Rogers, J. A. Materials and noncoplanar mesh designs for integrated circuits with linear elastic responses to extreme mechanical deformations. Proc. Nat Acad. Sci. USA 105, 18675 (2008).

$10 \mathrm{Ma}$, R., Menamparambath, M. M., Nikolaev, P. \& Baik, S. Transparent stretchable single-walled carbon nanotube-polymer composite films with near-infrared fluorescence. Adv. Mater. 25, 2548 (2013)

11 Xiao, L., Chen, Z., Feng, C., Liu, L., Bai, Z.-Q., Wang, Y., Qian, L., Zhang, Y., Li, Q. \& Jiang, K. Flexible, stretchable, transparent carbon nanotube thin film loudspeakers. Nano Lett. 8, 4539 (2008).

12 Yu, C., Masarapu, C., Rong, J., Wei, B. \& Jiang, H. Stretchable supercapacitors based on buckled single-walled carbon-nanotube macrofilms. Adv.Mater. 21, 4793 (2009).

$13 \mathrm{Ma}, \mathrm{R}$., Kwon, S., Zheng, A., Kwon, H. Y., Kim, J. I., Choi, H. R. \& Baik, S. Carbonnanotube/silver networks in nitrile butadiene rubber for highly conductive flexible adhesives. Adv. Mater. 24, 3344 (2012).

14 Moon, G. D., Lim, G. H., Song, J. H., Shin, M., Yu, T., Lim, B. \& Jeong, U. Highly stretchable patterned gold electrodes made of Au nanosheets. Adv. Mater. 25, 2707 (2013). 
15 Park, M., Im, J., Shin, M., Min, Y., Park, J., Cho, H., Park, S., Shim, M.-B., Jeon, S. \& Chung, D.-Y. Highly stretchable electric circuits from a composite material of silver nanoparticles and elastomeric fibres. Nat. Nanotechnol. 7, 803 (2012).

16 Wiley, B. J., Zang, J., Rathmell, A., Wu, J. \& Zhao, X. Reversible sliding in networks of nanowires. Nano Lett. 13, 2381-2386 (2013).

17 Lee, J. Lee, Lee, H., Yeo, J., Hong, S., Nam, K. H., Lee, D., Lee, S. S. \& Ko, S. H. Highly stretchable and highly conductive metal electrode by very long metal nanowire percolation network. Adv. Mater. 24, 3326 (2012).

$18 \mathrm{Xu}, \mathrm{F}$. \& Zhu, Y. Highly conductive and stretchable silver nanowire conductors. Adv. Mater. 24, 5117 (2012).

19 Akter, T. \& Kim, W. S. Reversibly stretchable transparent conductive coatings of spray-deposited silver nanowires. ACS Appl. Mater. Interfaces 4, 1855 (2012).

$20 \mathrm{Hu}$, W., Niu, X., Li, L., Yun, S., Yu, Z. \& Pei, Q. Intrinsically stretchable transparent electrodes based on silver-nanowire-crosslinked-polyacrylate composites. Nanotechnology 23, 344002 (2012).

21 Lee, M.-S., Lee, K., Kim, S.-Y., Lee, H., Park, J., Choi, K.-H., Kim, H. K., Kim, D.-G., Lee, D.-Y., Nam, S. \& Park, J.-U. High-performance, transparent, and stretchable electrodes using graphene-metal nanowire hybrid structures. Nano Lett. 13, 2814 (2013).

22 Wu, H., Hu, L., Rowell, M. W., Kong, D., Cha, J. J., McDonough, J. R., Zhu, J., Yang, Y., McGehee, M. D. \& Cui, Y. Electrospun metal nanofiber webs as high-performance transparent electrode. Nano. Lett. 10, 4242 (2010).

23 Wu, H., Kong, D., Ruan, Z., Hsu, P., Wang, S., Yu, Z., Carney, T. J., Hu, L., Fan, S. \& Cui, Y. A transparent electrode based on a metal nanotrough network. Nat. Nanotechnol. 8, 421-425 (2013).

24 Wu, J., Zang, J., Rathmell, A. R., Zhao, X. \& Wiley, B. J. Reversible sliding in networks of nanowires. Nano Lett. 13, 2381 (2013).

25 Jin, M., He, G., Zhang, H., Zeng, J., Xie, Z. \& Xia, Y. Shape-controlled synthesis of copper nanocrystals in an aqueous solution with glucose as a reducing agent and hexadecylamine as a capping agent. Angew. Chem. Inter. Ed. 50, 10560 (2011).

26 Won, Y., Kim, A., Lee, D., Yang, W., Woo, K., Jeong., S. \& Moon, J. Annealing-free fabrication of highly oxidation-resistive copper nanowire composite conductors for photovoltaics. NPG Asia Mater. 6, e105 (2014).
27 Woo, K., Kim, Y., Lee, B., Kim, J. \& Moon, J. Effect of carboxylic acid on sintering of inkjet-printed copper nanoparticulate films. ACS Appl. Mater. Interfaces 3, 2377 (2011).

$28 \mathrm{Kim}$, I. \& Kim, J. The effect of reduction atmospheres on the sintering behaviors of inkjet-printed Cu interconnectors. J. Appl. Phys. 108, 102807 (2010).

29 Sun, Y., Choi, W. M., Jiang, H., Huang, Y. Y. \& Rogers, J. A. Controlled buckling of semiconductor nanoribbons for stretchable electronics. Nat. Nanotechnol. 1, 201 (2006)

30 Chun, K.-Y., Oh, Y., Rho, J., Ahn, J.-H., Kim, Y.-J., Choi, H. R. \& Baik, S. Highly conductive, printable and stretchable composite films of carbon nanotubes and silver. Nat. Nanotechnol. 5, 853 (2010).

31 Jang, H. Y., Lee, S.-K., Cho, S. H., Ahn, J.-H. \& Park, S. Fabrication of metallic nanomesh: Pt nano-mesh as a proof of concept for stretchable and transparent electrodes. Chem. Mater. 25, 3535 (2013).

32 Ge, J., Yao, H. B., Wang, X., Ye, Y. D., Wang, J. L., Wu, Z. Y., Liu, J. W., Fan, F. J., Gao, H. L. \& Zhang, C. L. Stretchable conductors based on silver nanowires: improved performance through a binary network design. Angew. Chem. 125, 1698 (2013).

33 Shang, Y., He, X., Li, Y., Zhang, L., Li, Z., Ji, C., Shi, E., Li, P., Zhu, K., Peng, Q., Wang, C., Zhang, X., Wang, R., Wei, J., Wang, K., Zhu, H., Wu, D. \& Cao, A. Superstretchable spring-like carbon nanotube ropes. Adv. Mater. 24, 2896 (2012).

(c) (i) () $\Theta$ This work is licensed under a Creative Commons Attribution-NonCommercial-NoDerivs 3.0 Unported License. The images or other third party material in this article are included in the article's Creative Commons license, unless indicated otherwise in the credit line; if the material is not included under the Creative Commons license, users will need to obtain permission from the license holder to reproduce the material. To view a copy of this license, visit http://creativecommons.org/licenses/by-nc-nd/3.0/

Supplementary Information accompanies the paper on the NPG Asia Materials website (http://www.nature.com/am) 